\title{
25 Research Square \\ Identification and Screening of Novel Anti-Cancer Compound For Aurora Kinase A From Chemical Database
}

\section{Ipsa A. Singh}

Dr D Y Patil Vidyapeeth University Dr D Y Patil Biotechnology and Bioinformatics Institute Kiran Bharat Lokhande

Dr D Y Patil Vidyapeeth University Dr D Y Patil Biotechnology and Bioinformatics Institute

K. Venkateswara Swamy ( $\nabla$ venkateswara.swamy@mituniversity.edu.in )

MIT ADT University: MIT Art Design and Technology University https://orcid.org/0000-0001-8198-3042

\section{Research Article}

Keywords: Aurora kinase A, Fluoroflavones, chemoinformatics, Glide docking, MD simulation, Enrichment calculations

Posted Date: October 25th, 2021

DOI: https://doi.org/10.21203/rs.3.rs-924029/v1

License: (c) (i) This work is licensed under a Creative Commons Attribution 4.0 International License. Read Full License 


\section{Abstract}

Aurora kinase, a group of enzymes that belongs to a serine-threonine family and plays a critical role in cellular division. Aurora Kinase A is overexpressed and distributed beyond the nucleus and is involved in tumorigenesis. So, Aurora Kinase A is used as a drug target for cancer. Flavones are a class of flavonoids that are present in plants that show anticancer activity. Hence, the study will be on molecular binding modes between the derivative compounds of flavone-containing fluoro groups and the Aurora kinase A. Similar compounds of 2'Fluoroflavones are retrieved from the PubChem database. Then drug-like filters viz. REOS and PAINS were applied to remove toxic compounds using Canvas software. 3882 compounds are filtered, and 2449 compounds were obtained and are used as ligands with Aurora Kinase protein (PDB ID: 2J47) as a target, docking was done using Glide (Schrödinger) software. The lead compound among the above compounds was selected on the merit of hydrogen bonding, salt bridge as well as pi-pi interactions, 4-(6-Fluoro-4-oxychromen-2yl) benzoic acid has been found one of the best molecules from docking studies. The binding mode of the lead compound with AURKA reveals that the amino acid residues viz, Lys162, Ala213, and His280 are more important for binding, and having the best binding energy $(-11.760 \mathrm{kcal} / \mathrm{mol})$ was noted among the ligand datasets. Then resulted Enrichment calculations is suggesting that the docking protocol is validated because the Receiver operating characteristic curve (ROC) curve shows $\mathrm{R}^{2}=0.99 .100 \mathrm{~ns}$ MD simulations of Aurora Kinase $A$ and lead compounds confirm thermodynamic stability as compared to standard compounds. The molecular dynamics simulations of 100 ns were done, which shows that the mean RMSD value of $1.77 \AA$ for all 3 complexes of the protein and Fluoroflavone and its analogs. This shows that Fluoroflavone and its 2 best analogs are tightly attached to the active sites and thus having conformational stability. Our finding suggests that 4-(6fluoro-4-oxochromen-2-yl)benzoic acid and 4-(4-0xochromen-2-yl)benzoate can be further used in vitro and in vivo experiments and can probably serve as a novel drug for cancer treatment.

\section{Introduction}

Aurora Kinases are a group of enzymes that belong to the serine-threonine family. They play a major role in cell division by controlling chromatid segregation during different phases of the cell cycle [1]. These Kinases play an important role in the distribution of various steps of mitotic changes. They play important role in maintaining the role of mitosis and thus making it an attractive novel target for cancer treatment [2]. The function of Aurora Kinase $A$ is to duplicate centrosome at the start of the $S$ phase and slowly spindle microtubules move during mitosis to the nuclear material of the daughter cells at the end of the mitosis (Table 1) [3]. It acts as a regulative part of the p53/TP53 pathway, and peculiarly at the checkpoint-response pathways is more important for changes of oncogenic alterations, by phosphorylating and maintaining p53/TP53 $[4,5]$. Recent studies revealed that Aurora Kinase A helps in tumor development by the stimulation of epithelial-mesenchymal change, which results in the genesis of tumor cells [6]. Therefore, Aurora kinase A being overexpressed has been linked to oncogenic changes mainly linked to centrosome amplification [7]. Thus, Aurora kinase A plays a " druggable target" in cancer, which controls oncogenic pathways associated with drug resistance [8, 9]. We have targeted Aurora 
kinase A with PDB ID: 2 J4Z (Fig. 1) with small natural inhibitors with fewer side effects to inhibit tumor progression [10-12].

The backbone structure of flavones which belong to the class flavonoids is 2-phenylchromen-4-one(2phenyl-1-benzopyran-4-one) (Fig. 2) [13-17]. Flavones are generally derived from natural sources, i.e. from fruits and plants. Some of the flavones show anticancer properties and can be used in cancer therapy. On the other hand, the structural properties of flavones that show inhibitory effects on cancerous cell growth remain not clear [18-20]. To make out the structural properties of flavone, which exhibit the inhibition of cancer cells, we retrieve Fluoroflavone derivatives as inhibitors from the database for Aurora kinase A protein. The binding of the Fluoroflavones and their analogs with Aurora kinase A can be calculated by in silico docking experiments. This provides the knowledge for designing novel anticancer drugs that target Aurora Kinase A protein.

\section{The Hypothesis}

Knowing the fact that overexpression of the Aurora Kinase A causes different types of cancer. To stop overexpression of Aurora Kinase, we have hypothesized that Fluoroflavone and its analogs can be the inhibitor. The present work investigates that Aurora Kinase A protein is inhibited by Fluoroflavone and its analogs using the computational approach of molecular drug designing and molecular dynamics.

\section{Evaluation of Hypothesis}

\section{Materials And Methodology}

Protein crystal structure preparation

Three-dimensional (3D) crystal structures of (PDB ID:2J4Z) Aurora Kinase A protein was retrieved from RCSB Protein Data Bank. The protein retrieved (PDB ID:2J4Z) has resolutions of 2.0Å. Before performing the docking calculations, the crystal structures of Aurora kinase $A$ were prepared at $\mathrm{pH} 7 \pm 1$ by the PROPKA program and used protein preparation wizard of Schrodinger (Protein Preparation Wizard: Schrodinger Release 2019-2). The OPLS3e force field was used to optimize the crystal structure and the side chains.

Filtration

Fluoroflavone derivative of flavones (CID: 261400) and its compounds similar (90\% similar) are taken as inhibitors against Aurora Kinase A. 3882 compounds were obtained from PubChem database (https://pubchem.ncbi.nlm.nih.gov/). These compounds are further filtered using different methods. Firstly, these compounds were pushed to Entrez and a filter of Lipinski's rule of 5 was added and 3882 compounds were filtered in we have 3430 compounds. There are different filters of CANVAS of Schrodinger 2019-software, which filter compounds based on different parameters. REOS is one of the filters which sets the criteria to identify lead-like small compounds and the compounds are filtered. Filtering 3430 compounds to 2449 compounds. The next filter of CANVAS is PAINS. PAIN filter 
compounds with the substructure. PAINS1, PAINS2, and PAINS3 filtered 2449 compounds to 2448 compounds.

Ligand structure retrieval and its preparation

Derivatives of Fluoroflavone were filtered and are used as an inhibitor to inhibit the overexpression of Aurora Kinase A. The 2 dimensional (2D) chemical structures of Fluoroflavone (PubChem CID: 261400) and its derivatives were retrieved from the PubChem database and was filtered. LigPrep (Schrodinger Release 2019-2) is used for the 2D structures of the ligands and was subjected to energy minimization and appropriate bond order assignment with Optimized Potentials for Liquid Simulations (OPLS3e) force field (module of Schrodinger to get a stable conformation of the analogs of the Fluoroflavone).

\section{Molecular docking}

The binding cavities of targeted receptors were defined considering all residues which come within the cut off distance of $2 \AA$ from the crystal ligands which are present in the retrieved crystal structure of Aurora kinase A receptor and also, the binding cavity information for this receptor was taken from PDBsum (www.ebi.ac.uk/pdbsum/). The prepared 2448 ligands (filtered analogs of Fluoroflavone) were subjected to docking calculations within the defined binding cavity using the Extra Precision (XP) and Standard Precision (SP) different modes of Glide program (Glide: Schrodinger Release 2019-2) with the OPLS3e force field. Aurora Kinase A receptor, and binding cavity of Aurora Kinase A protein forms a complex structure of the ligands and the protein by docking into the binding cavity using SP and XP mode of Glide [21]. We further extended the data to understand the interactions of residues with ligands.

\section{Enrichment calculations}

"Enrichment" is the measure of known ligands ranks to a set of decoys. Enrichments of ligands is required to improve the binding affinity with specific residues in the cavity of targeted Kinase [22] using DUD.E database decoys was designed for decoy bias on enrichment. For Aurora Kinase A protein, a decoy set for Aurora Kinase is generated using the DUD.E database [23]. Decoys are computed on the basis of similar physical properties but different chemical structures. So active set of decoys are generated, but they have not been tested. So, the random 1000 drug-like decoys for Aurora Kinase and 10 best XP docked ligands and Fluoroflavone molecules were docked with Aurora Kinase A (PDB ID: 2J4Z). A module for calculating Enrichment Factors and the effectiveness of ligands and decoys with target protein was calculated using the Enrichment Schrodinger analysis enrichment calculator module.

Molecular dynamic (MD) studies

Molecular dynamic studies are used to evaluate the strength to bind and activity of Fluoroflavone, and the best 2 analogs of Fluoroflavone were obtained after docking studies. The full-scale molecular dynamic simulation was performed for 100ns with a complex of Fluoroflavone and its 2 best analogs of Fluoroflavone with Aurora Kinase A. The process of Molecular dynamic simulations using Desmond helps to measure the movements of the atoms and calculate the forces. However, Desmond considers all 
detailed requirements including temperature, pressure, volume system, and all the functionality used to study the complex interactions. System builder of Desmond in the Maestro program is used, the system for the protein-ligand complex is immersed in a water-filled cubic box containing $1 \AA$ spacing water molecules using an extended TIP3 (three-point water model) with periodic boundary conditions. The solvate complex system is charged and is neutralized by adding counter ions randomly. The steepest descent method is used for energy minimization which is an important step for MD simulations. To apply boundary conditions to the protein-ligand complex, a cube box (box size 8.0) is taken for reducing the edge effects in the finite system. The total system is surrounded by translated copies of itself and the atoms are in the space-filling box. The OPLS3e force field (parameters used to describe the potential energy of a system) is chosen, which has an improved force field for MD simulation of proteins [24]. Certain parameters as input such as constraints set as all-bonds, integrator as MD noise is considered in the studies of molecular dynamics. However, the chain thermostat method and it uses the MartynaTobias-Klein barostat method at a temperature of 300k. After the system gains the state of equilibrium, the stable conformation trajectories are taken into account and analyzed to inspect interactions and stability. The C-alpha backbone's, conformational changes of Aurora Kinase A crystal structure have been compared with previous conformations. Besides, we have performed MD trajectory clustering analysis to find out the binding mode of the selected analogs of the Fluoroflavone within the active sites of the protein using trajectories generated during 100ns Molecular Dynamic simulations.

MD trajectory analysis and prime MM/GBSA calculations

The binding free energy of the complex system was calculated using the Prime module. To calculate the ligand binding free energies and ligand strain energies for the complexes of Aurora Kinase $A$ with the ligands, the MM/GBSA (Molecular Mechanics, The Generalized Born Model, and Solvent Accessibility) was used. The non-polar solvation energies, polar solvation energies, and potential energy are comprised of binding free energy. Prime MM-GBSA works with the combination of advanced OPLS-3E force field, SGB solvation model for polar solvation (GSGB), non-polar solvation (GNP), and Molecular Mechanics Energies (EMM) that compiled different nonpolar solvent accessible surface area and van der Waals interactions. The free energy changes upon ligand binding were calculated using the following equations.

$\Delta$ Gbind $=$ Gcomplex $-($ Gprotein + Gligand $) \mathrm{G}=\mathrm{EMM}+\mathrm{GSGB}+\mathrm{GNP}$.

The $G_{\text {complex }}$ represents complex energy, $G_{\text {protein }}$ is the receptor energy and $G_{\text {ligand }}$ is the unbound ligand energy. EMM represents molecular mechanics energies, GSGB is an SGB solvation model for polar solvation and GNP is a nonpolar solvation term [25].

\section{Results And Discussion}

Molecular Docking Analysis

The molecular docking analysis of the Aurora Kinase A, with the flavone derivative (f Fluoroflavone) and its 2448 analogs compounds were docked using SP and XP Glide. The best 5 docked SP glide 
calculations are reported in Table 2 and the best 5 of XP glide calculation reported in Table 3. The results of docking simulations show the best 5 ligands binding to the active site of the Aurora A kinase and its interactions i.e., hydrogen bonds, aromatic hydrogen bond, salt bridges, and the pi-pi and its bond length were observed and which thus shows the binding of the ligands with the proteins. The intermolecular interactions of standard compound i.e., Fluoroflavone with Aurora Kinase A shown in Fig. 3, shows that the Fluoroflavone interacted with Aurora Kinase A by forming one hydrogen bond and one pi-pi stacking with amino acid Ala213 at bond distances of $2.16 \AA$ and $2.50 \AA$ respectively. The lead compound (CID 82043699) i.e., 4-(6-Fluoro-4-oxychromen-2yl) benzoic acid (Fluoroflavone Analog 1) after SP docking gives the best binding energy score of $-10.059 \mathrm{kcal} / \mathrm{mol}$ out of 2449 compounds and forms a hydrogen bond with Ala213 and Lys162 at a bond distance of $1.85 \AA$ and $2.01 \AA$ (Fig. 4) respectively. The aromatic hydrogen atom interaction with Ala 213 at a bond distance of $3.13 \AA$, salt bridges with Lys 162 with a bond length of $2.72 \AA$ and pi-pi- stacking interactions with His 280 at the bond length of $5.36 \AA$. Ligand with Fluoroflavone Analog 1 after XP docking with Aurora kinase A also gives the best docking score i.e., binding energy $-11.760 \mathrm{kcal} / \mathrm{mol}$. There were different interactions, hydrogen atom interactions were with Ala213 and Lys 162 with $1.86 \AA$ and $2.00 \AA$ respectively (Fig. 4). The aromatic $\mathrm{H}$ bond interactions were with Ala213 and Tyr212 with bond lengths of 2.50 and $2.89 \AA$ respectively, the salt bridge interactions with Lys 162 with a bond length of $2.72 \AA$, and the pi-pi stacking with a molecule with His 280 with bond length $\AA$.

From the molecular docking studies, stable binding interactions were observed for both the docking modules i.e. SP and XP. The best-docked ligand out of 2449 ligands is the same but slightly varies in binding energy and interactions. The Binding energy for the XP module gives better scores and slightly different interactions as compared to SP. Thus, we conclude from the interactions that the compound Fluoroflavone Analog 1 shows the best docking results. The next best lead compound Fluoroflavone Analog 2 (CID: 7728977) shown in Fig. 5 i.e., 4-(4-oxochromen-2-yl) benzoate.

\section{Enrichment Calculations}

The mapping of the 10 best-docked analogs of Fluoroflavone, Fluoroflavone and 1000 decoys for Aurora Kinase had been docked and enrichment calculations were done. The results of enrichment calculations suggest that docking protocol is validated because Receiver Operating Curve (ROC) shows $R^{2}=0.99$. The enrichment graph (Fig. 6) shows that the 10 best ligands and reference molecule shows better results as compared to the decoy sets. Thus, we validate from the graph and the results the Fluoroflavone analogs (best 10 docked analogs of Fluoroflavone and the Fluoroflavone) show a better potential drug for Aurora Kinase A compared to the decoys for Aurora Kinases. Enrichment further validated our docking results.

Molecular dynamic (MD) studies

Molecular docking and enrichment calculations were done on the rigid crystal structure of the protein, and interactions between the Fluoroflavone and its analogs using molecular dynamic simulation to study the stability of the ligands within the active sites of the crystal structure of the Aurora Kinase A protein. To 
study the stability of the complexes and their different rearrangements of Ca atoms of Aurora Kinase A complexes with the Fluoroflavone and its analogs.

Thermodynamic conformation stability for 100 ns is studied using RMSD and RMSF (Root values of C-a atoms of Aurora Kinase A. Later, 1000 trajectories are being formed during the Molecular Dynamic simulations. Each trajectory is superimposed to the initial position of Aurora Kinase A. The complex of Aurora Kinase A uses the Simulation Event Analysis (SEA) panel of Schrodinger and the output file obtained in ".dat" format. This data of RMSF and RMSD values which are collected were used to plot graphs for RMSD and RMSF. The RMSD plot (Fig. 7) suggests that Fluoroflavone, Fluoroflavone Analog 1 , and Fluoroflavone Analog 2 are within the good range of RMSD which is acceptable where the mean RMSD value is $1.77 \AA$, which indicates that the binding of the protein and the ligands is good. The RMSD graph (Fig. 8) shows that the complexes started gaining equilibrium from $35 \mathrm{~ns}$ to $100 \mathrm{~ns}$ within the range of RMSD $1.3 \AA$ to $2.4 \AA$, which gives the average RMSD value of $1.77 \AA$

Prime MM/GBSA energies for the complexes of Aurora Kinase A protein

Free binding energies ( $\triangle \mathrm{G}$ Bind) are calculated by MM/GBSA. MM/GBSA states the results in terms of solvation components or VDW, hydrophobic. The Fluoroflavone, Fluoroflavone Analog 1, and Fluoroflavone Analog 2 (CID: 261400, 82043699, 7728977), serving as ligands to Aurora Kinase A protein were submitted to ensemble-averaged Prime MM/GBSA method for a long time MD simulation. The ensemble-average of binding free energies of the 3 complexes of protein-ligand are reported in Table 4. The binding energy is less (more negative value) which means that the complex is stronger. The data denotes that compound with CID 82043699 and compound with CID 7728977 both have the most negative binding free energy $(-50.898 \pm 3.073$ and $52.87 \pm 1.8773)$ which confers the binding stability of both the analogs to be more potent. Whereas another reference compound also shows favorable binding free energy with Aurora Kinase A protein, suggesting that these 3 molecules make a strong complex with the active site of Aurora Kinase A. These MM/GBSA results suggest that the ligands satisfy the prime MM/GBSA approach to achieve a stable complex with Aurora Kinase A protein. The free energies predicted from the prime MM/GBSA show that complexes are thermodynamically favorable.

\section{Conclusion}

Aurora Kinase A plays an important role in the cell cycle, overexpression of the protein can cause different types of cancer. Thus, we can use Aurora Kinase A as target protein against analogs of Fluoroflavone, as flavones are the natural derivatives. Thus, in this research work, we used the strategy we take analogs of Fluoroflavone which are $90 \%$ close to Fluoroflavone. The library of 3882 analogs of Fluoroflavone is used and filtered using many methods. After filtration, filtered analogs (2448 compounds) were used to perform molecular docking with Aurora Kinase A by using glide software. The result obtained shows that 4-(6-fluoro-4-oxochromen-2-yl)benzoic acid shows the best docking score of $-11.760 \mathrm{kcal} / \mathrm{mol}$ among all the analogs docked. 
MD simulations were carried out for 100 ns for each of these three complexes of Aurora Kinase $A$ and Fluoroflavone, Fluoroflavone Analog1, and Fluoroflavone Analog 2 with the mean RMSD value of $1.77 \AA$. The molecular dynamics results further confer the conformational stability of the protein-ligand complexes as the RMSF of the pocket residues after binding of the ligand and the protein is lower than $2.0 \AA$. Also, the MM/GBSA free energy calculations reveal that the analog compounds form a stable complex with Aurora Kinase A. Thus, the obtained Fluoroflavone Analog 1 and Fluoroflavone Analog 2 can be further used in vitro and in vivo experiments and can probably serve as a novel drug for cancer treatment.

\section{Declarations}

\section{Acknowledgment:}

The authors are thankful to Dr. D. Y. Patil Biotechnology and Bioinformatics Institute Dr. D. Y. Patil Vidyapeeth Tathawade, Pune, for providing the infrastructure and facilities to perform this study. The authors also acknowledge the DST-SERB, Govt. of India, New Delhi, (File Number: YSS/2015/002035) for Optimized Supercomputer facility for dynamics calculations. Mr. Kiran Bharat Lokhande acknowledges the ICMR (Indian Council of Medical Research), New Delhi, India for Senior Research Fellowship (Project ID: 2019-3458; file: ISRM/11(54)/2019). The authors also would like to acknowledge the support of Schrodinger, and the team for providing an Evaluation License to our Bioinformatics Research Laboratory.

Funding: Not applicable.

Conflicts of interest/Competing interests: The author declares no conflict of interest.

Consent statement/Ethical approval: Not applicable.

Availability of data and material: The source of data and materials have been mentioned in the manuscript, in support of the findings.

Code availability: Not applicable.

Authors' contributions: K. Venkateswara Swamy: Conceptualization, Supervision, Software, Data Curation, Review; Ipsa A. Singh: Analysis Methodology, Writing - Original Draft, Visualization, Resources; Kiran Bharat Lokhande: Supervision Validation, Writing - Review and Editing.

\section{References}

1. Ma HT, Poon R (2020) Aurora kinases and DNA damage response. Mutat Res 821:111716. https://doi.org/10.1016/j.mrfmmm.2020.111716

2. Tang A, Gao K, Chu L, Zhang R, Yang J, Zheng J (2017) Aurora kinases: novel therapy targets in cancers. Oncotarget 8(14):23937-23954. https://doi.org/10.18632/oncotarget.14893 
3. Carvalhal S, Ribeiro SA, Arocena M, Kasciukovic T, Temme A, Koehler K, Huebner A, Griffis ER (2015) The nucleoporin ALADIN regulates Aurora A localization to ensure robust mitotic spindle formation. Molecular biology of the cell 26(19):3424-3438. https://doi.org/10.1091/mbc.E15-02-0113

4. Kollareddy M, Dzubak P, Zheleva D, Hajduch M (2008) Aurora kinases: structure, functions and their association with cancer. Biomedical papers of the Medical Faculty of the University Palacky, Olomouc, Czechoslovakia, 152(1), 27-33. https://doi.org/10.5507/bp.2008.004

5. Kamran M, Long ZJ, Xu D, Lv SS, Liu B, Wang CL, Xu J, Lam EW, Liu Q (2017) Aurora kinase A regulates Survivin stability through targeting FBXL7 in gastric cancer drug resistance and prognosis. Oncogenesis 6(2):e298. https://doi.org/10.1038/oncsis.2016.80

6. Takahashi, Y., Sheridan, P., Niida, A., Sawada, G., Uchi, R., Mizuno, H., Kurashige,J., Sugimachi, K., Sasaki, S., Shimada, Y., Hase, K., Kusunoki, M., Kudo, S., Watanabe,M., Yamada, K., Sugihara, K., Yamamoto, H., Suzuki, A., Doki, Y., Miyano, S., ... Mimori,K. (2015). The AURKA/TPX2 axis drives colon tumorigenesis cooperatively with MYC. Annals of oncology: official journal of the European Society for Medical Oncology, 26(5), 935-942. https://doi.org/10.1093/annonc/mdv034

7. Nguyen AL, Drutovic D, Vazquez BN, Yakoubi E, Gentilello W, Malumbres AS, Solc M, P., \& Schindler K (2018) Genetic Interactions between the Aurora Kinases Reveal New Requirements for AURKB and AURKC during Oocyte Meiosis. Current biology: CB 28(21):3458-3468.e5. https://doi.org/10.1016/j.cub.2018.08.052

8. Malumbres M, Pérez de Castro, I (2014) Aurora kinase A inhibitors: promising agents in antitumoral therapy. Expert Opin Ther Targets 18(12):1377-1393. https://doi.org/10.1517/14728222.2014.956085

9. D'Assoro AB, Haddad T, Galanis E. Aurora-A Kinase as a Promising Therapeutic Target in Cancer. Front Oncol. 2016 Jan 6;5:295. doi: 10.3389/fonc.2015.00295. PMID: 26779440; PMCID: PMC4701905

10. Matthews N, Visintin C, Hartzoulakis B, Jarvis A, Selwood DL (2006) Aurora A and B kinases as targets for cancer: will they be selective for tumors? Expert Rev Anticancer Ther 6(1):109-120. https://doi.org/10.1586/14737140.6.1.109

11. Shin SY, Lee Y, Kim BS, Lee J, Ahn S, Koh D, Lim Y, Lee YH (2018) Inhibitory Effect of Synthetic Flavone Derivatives on Pan-Aurora Kinases: Induction of G2/M Cell-Cycle Arrest and Apoptosis in HCT116 Human Colon Cancer Cells. Int J Mol Sci 19(12):4086. https://doi.org/10.3390/ijms19124086

12. Xie Y, Zhu S, Zhong M, Yang M, Sun X, Liu J, Kroemer G, Lotze M, Zeh HJ 3rd, Kang R, Tang D (2017) Inhibition of Aurora Kinase A Induces Necroptosis in Pancreatic Carcinoma. Gastroenterology 153(5):1429-1443.e5. https://doi.org/10.1053/j.gastro.2017.07.036

13. Yan $M$, Wang C, He B, Yang M, Tong M, Long Z, Liu B, Peng F, Xu L, Zhang Y, Liang D, Lei H, Subrata S, Kelley KW, Lam EW, Jin B, Liu Q (2016) Aurora-A Kinase: A Potent Oncogene and Target for Cancer Therapy. Medicinal research reviews 36(6):1036-1079. https://doi.org/10.1002/med.21399 
14. Lee YH, Park J, Ahn S, Lee Y, Lee J, Shin SY, Koh D, Lim Y (2019) Design, synthesis, and biological evaluation of polyphenols with 4,6-diphenylpyrimidin-2-amine derivatives for inhibition of Aurora kinase A. Daru: journal of Faculty of Pharmacy Tehran University of Medical Sciences 27(1):265281. https://doi.org/10.1007/s40199-019-00272-5

15. Long L, Wang YH, Zhuo JX, Tu ZC, Wu R, Yan M, Liu Q, Lu G (2018) Structure-based drug design: Synthesis and biological evaluation of quinazolin-4-amine derivatives as selective Aurora A kinase inhibitors. Eur J Med Chem 157:1361-1375. https://doi.org/10.1016/j.ejmech.2018.08.053

16. Katayama H, Sen S (2010) Aurora kinase inhibitors as anticancer molecules. Biochim Biophys Acta 1799(10-12):829-839. https://doi.org/10.1016/j.bbagrm.2010.09.004

17. Jung Y, Shin SY, Yong Y, Jung H, Ahn S, Lee YH, Lim Y (2015) Plant-derived flavones as inhibitors of aurora $B$ kinase and their quantitative structure-activity relationships. Chem Biol Drug Des 85(5):574-585. https://doi.org/10.1111/cbdd.12445

18. Libertini S, Abagnale A, Passaro C, Botta G, Portella G (2010) Aurora A and B kinases-targets of novel anticancer drugs. Recent Pat Anti-cancer Drug Discov 5(3):219-241.

https://doi.org/10.2174/157489210791760517

19. Ma HT, Poon R (2020) Aurora kinases and DNA damage response. Mutat Res 821:111716. https://doi.org/10.1016/j.mrfmmm.2020.111716

20. van Gijn SE, Wierenga E, van den Tempel N, Kok YP, Heijink AM, Spierings D, Foijer F, van Vugt M, Fehrmann R (2019) TPX2/Aurora kinase A signaling as a potential therapeutic target in genomically unstable cancer cells. Oncogene 38(6):852-867. https://doi.org/10.1038/s41388-018-0470-2

21. Lokhande KB, Doiphode S, Vyas R, Swamy KV (2020) Molecular docking and simulation studies on SARS-CoV-2 $\mathrm{M}^{\text {pro }}$ reveals Mitoxantrone, Leucovorin, Birinapant, and Dynasore as potent drugs against COVID-19. J Biomol Struct Dyn, 1-12. Advance online publication. https://doi.org/10.1080/07391102.2020.1805019

22. Mysinger MM, Carchia M, Irwin JJ, Shoichet BK (2012) Directory of useful decoys, enhanced (DUDE): better ligands and decoys for better benchmarking. Journal of medicinal chemistry 55(14):65826594. https://doi.org/10.1021/jm300687e

23. Chaput L, Martinez-Sanz J, Saettel N, Mouawad L (2016) Benchmark of four popular virtual screening programs: construction of the active/decoy dataset remains a major determinant of measured performance. J Cheminform 8:56. https://doi.org/10.1186/s13321-016-0167-x

24. Lokhande KB, Apte GR, Shrivastava A, Singh A, Pal JK, Venkateswara Swamy K, Gupta RK (2020) Sensing the interactions between carbohydrate-binding agents and $N$-linked glycans of SARS-CoV-2 spike glycoprotein using molecular docking and simulation studies. J Biomol Struct Dyn, 1-19. Advance online publication. https://doi.org/10.1080/07391102.2020.1851303

25. Mansuri A, Lokhande K, Kore S, Gaikwad S, Nawani N, Swamy KV, Junnarkar M, Pawar S (2020) Antioxidant, anti-quorum sensing, biofilm inhibitory activities and chemical composition of Patchouli essential oil: in vitro and in silico approach. J Biomol Struct Dyn, 1-12. Advance online publication. https://doi.org/10.1080/07391102.2020.1810124 


\section{Tables}

Table 1

Location, function, and tumor types in different Aurora kinases.

\begin{tabular}{|c|c|c|c|}
\hline Kinases & Localization & Function & Tumor type \\
\hline $\begin{array}{l}\text { Aurora } \\
\text { Kinase } \\
\text { A }\end{array}$ & $\begin{array}{l}\text { Midbody, } \\
\text { Spindle } \\
\text { microtubule } \\
\text { Centrosome }\end{array}$ & $\begin{array}{l}\text { Centrosome maturation and } \\
\text { separation, Microtubule } \\
\text { nucleation, Bipolar spindle } \\
\text { microtubule formation, } \\
\text { Cytokinesis, Mitosis exit, } \\
\text { Mitotic entry, Spindle } \\
\text { assembly }\end{array}$ & $\begin{array}{l}\text { Ovarian cancer, Oral cancer, } \\
\text { Gastric/Gastrointestinal cancer, } \\
\text { Esophageal squamous cell carcinoma, } \\
\text { Cervical cancer, Prostate cancer, Glioma, } \\
\text { Acute myeloid leukemia, Lung cancer, } \\
\text { Breast cancer, Colorectal cancer }\end{array}$ \\
\hline
\end{tabular}


Table 2

Detailed intermolecular interactions of Fluoroflavone and its derivatives with Aurora Kinase A (SP docking results).

\begin{tabular}{|c|c|c|c|c|}
\hline Compound ID & Binding Energy (kcal/mol) & Interaction & Bond Type & Bond length ( $\AA$ ) \\
\hline \multirow[t]{5}{*}{82043699} & \multirow[t]{5}{*}{-10.059} & Ala213 & $\mathrm{H}$-bond & 1.85 \\
\hline & & Ala213 & Aromatic $\mathrm{H}$-Bond & 3.13 \\
\hline & & Lys 162 & $\mathrm{H}$-bond & 2.01 \\
\hline & & Lys 162 & Salt Bridge & 2.72 \\
\hline & & His280 & Pi-Pi Stacking & 5.36 \\
\hline \multirow[t]{3}{*}{29947976} & \multirow[t]{3}{*}{-9.976} & Ala213 & $\mathrm{H}$-bond & 1.94 \\
\hline & & Ala213 & Aromatic $\mathrm{H}$-Bond & 2.39 \\
\hline & & Tyr212 & Aromatic $\mathrm{H}$-bond & 2.92 \\
\hline \multirow[t]{6}{*}{11369667} & \multirow[t]{6}{*}{-9.887} & Ala213 & $\mathrm{H}$-Bond & 1.88 \\
\hline & & Ala213 & Aromatic $\mathrm{H}$-Bond & 2.52 \\
\hline & & Tyr212 & Aromatic $\mathrm{H}$-Bond & 2.90 \\
\hline & & Glu-260 & Aromatic $\mathrm{H}$-Bond & 2.74 \\
\hline & & Val147 & Aromatic $\mathrm{H}$-Bond & 4.65 \\
\hline & & His280 & Pi-Pi Stacking & 3.71 \\
\hline \multirow[t]{5}{*}{7728977} & \multirow[t]{5}{*}{-9.886} & Lys162 & $\mathrm{H}$-bond & 2.01 \\
\hline & & Ala213 & $\mathrm{H}$-bond & 1.86 \\
\hline & & Ala213 & Aromatic $\mathrm{H}$-Bond & 2.97 \\
\hline & & Lys162 & Aromatic $\mathrm{H}$-Bond & 2.51 \\
\hline & & His280 & Pi-Pi Stacking & 5.35 \\
\hline \multirow[t]{6}{*}{122404136} & \multirow[t]{6}{*}{-9.671} & Ala213 & $\mathrm{H}$-bond & 1.93 \\
\hline & & Tyr212 & Aromatic $\mathrm{H}$-bond & 2.93 \\
\hline & & Ala213 & Aromatic $\mathrm{H}$-bond & 2.77 \\
\hline & & Glu-260 & Aromatic $\mathrm{H}$-bond & 2.71 \\
\hline & & Ala273 & Aromatic $\mathrm{H}$-bond & 2.77 \\
\hline & & His280 & Pi-Pi Stacking & 4.07 \\
\hline
\end{tabular}




\begin{tabular}{|lllll|}
\hline Compound ID & Binding Energy $(\mathrm{kcal} / \mathrm{mol})$ & Interaction & Bond Type & Bond length $(\AA)$ \\
\hline 261400 & -8.752 & Ala213 & H-bond & 2.16 \\
\cline { 2 - 5 } & & Ala213 & Pi-Pi Stacking & 2.50 \\
\hline
\end{tabular}


Table 3

Detailed intermolecular interactions of Fluoroflavone and its derivatives with Aurora Kinase A (XP docking results).

\begin{tabular}{|c|c|c|c|c|}
\hline Compound ID & $\begin{array}{l}\text { Binding Energy } \\
\text { (kcal/mol) }\end{array}$ & Interaction & Bond Type & $\begin{array}{l}\text { Bond length } \\
(\AA)\end{array}$ \\
\hline \multirow[t]{6}{*}{82043699} & \multirow[t]{6}{*}{-11.760} & Ala213 & $\mathrm{H}$-bond & 1.86 \\
\hline & & Lys162 & $\mathrm{H}$-bond & 2.00 \\
\hline & & Lys162 & Salt Bridge & 2.97 \\
\hline & & Ala213 & Aromatic $\mathrm{H}$-bond & 2.50 \\
\hline & & Tyr212 & Aromatic $\mathrm{H}$-bond & 2.89 \\
\hline & & His280 & Pi-Pi Stacking & 5.36 \\
\hline \multirow[t]{6}{*}{7728977} & \multirow[t]{6}{*}{-11.645} & Ala213 & $\mathrm{H}$-bond & 1.88 \\
\hline & & Lys162 & $\mathrm{H}$-bond & 1.65 \\
\hline & & Lys162 & Salt Bridge & 4.16 \\
\hline & & Ala213 & Aromatic $\mathrm{H}$-bond & 2.40 \\
\hline & & Tyr212 & Aromatic $\mathrm{H}$-bond & 2.90 \\
\hline & & His280 & Aromatic $\mathrm{H}$-bond & 2.95 \\
\hline \multirow[t]{3}{*}{29947976} & \multirow[t]{3}{*}{-10.096} & Ala213 & $\mathrm{H}$-bond & 1.94 \\
\hline & & Ala213 & Aromatic $\mathrm{H}$-bond & 2.46 \\
\hline & & Tyr212 & Aromatic $\mathrm{H}$-bond & 2.93 \\
\hline \multirow[t]{4}{*}{461719627} & \multirow[t]{4}{*}{-9.929} & Ala213 & $\mathrm{H}$-bond & 2.04 \\
\hline & & Ala213 & Aromatic $\mathrm{H}$-bond & 2.22 \\
\hline & & Tyr212 & Aromatic $\mathrm{H}$-bond & 3.21 \\
\hline & & Glu211 & Aromatic $\mathrm{H}$-bond & 2.12 \\
\hline \multirow[t]{3}{*}{70513520} & \multirow[t]{3}{*}{-9.900} & Ala213 & $\mathrm{H}$-bond & 1.83 \\
\hline & & Ala213 & Aromatic $\mathrm{H}$-bond & 2.45 \\
\hline & & Tyr212 & Aromatic $\mathrm{H}$-bond & 2.88 \\
\hline
\end{tabular}


Table 4

The ensemble-averaged prime binding free energies $(\mathrm{kcal} / \mathrm{mol})$ of docked complexes during $100 \mathrm{~ns} \mathrm{MD}$ simulation.

\begin{tabular}{|c|c|c|c|c|c|}
\hline $\begin{array}{l}\text { Floro flavone } \\
\text { derivatives (CID) }\end{array}$ & $\begin{array}{l}\Delta G \text { Bind }{ }^{1} \\
(\mathrm{kcal} / \mathrm{mol})\end{array}$ & $\begin{array}{l}\Delta G \text { Coulomb } \\
(\mathrm{kcal} / \mathrm{mol})\end{array}$ & $\begin{array}{l}\Delta G \text { Bind } \\
\mathrm{vdW}^{3} \\
(\mathrm{kcal} / \mathrm{mol})\end{array}$ & $\begin{array}{l}\Delta G \text { Solv } \\
\mathrm{GB}^{4} \\
(\mathrm{kcal} / \mathrm{mol})\end{array}$ & $\begin{array}{l}\text { Complex } \\
\text { Energy }^{5} \\
\text { (kcal/mol) }\end{array}$ \\
\hline Fluoroflavone & $\begin{array}{l}-47.172 \pm \\
2.44\end{array}$ & $-5.110 \pm 2.25$ & $\begin{array}{l}-31.790 \pm \\
2.21\end{array}$ & $\begin{array}{l}8.239 \pm \\
1.59\end{array}$ & $\begin{array}{l}-7588.120 \\
\pm 53.72\end{array}$ \\
\hline $\begin{array}{l}\text { Fluoroflavone Analog } \\
1\end{array}$ & $\begin{array}{l}-50.898 \pm \\
3.07\end{array}$ & $-67.690 \pm 4.75$ & $\begin{array}{l}-34.735 \pm \\
2.32\end{array}$ & $\begin{array}{l}70.956 \pm \\
3.02\end{array}$ & $\begin{array}{l}-7713.268 \\
\pm 79.21\end{array}$ \\
\hline $\begin{array}{l}\text { Fluoroflavone Analog } \\
2\end{array}$ & $\begin{array}{l}-52.87 \pm \\
1.87\end{array}$ & $-60.682 \pm 5.23$ & $\begin{array}{l}-37.679 \pm \\
2.27\end{array}$ & $\begin{array}{l}66.172 \pm \\
3.44\end{array}$ & $\begin{array}{l}-7655.969 \\
\pm 45.05\end{array}$ \\
\hline
\end{tabular}

${ }^{1} \mathrm{MM} / \mathrm{GBSA}$ binding free energy.

${ }^{2}$ Coulomb energy.

${ }^{3}$ Van der Waals energy.

${ }^{4} \mathrm{~GB}$ Generalized Born electrostatic solvation energy.

${ }^{5}$ Energy of protein-ligand complex

Figures 


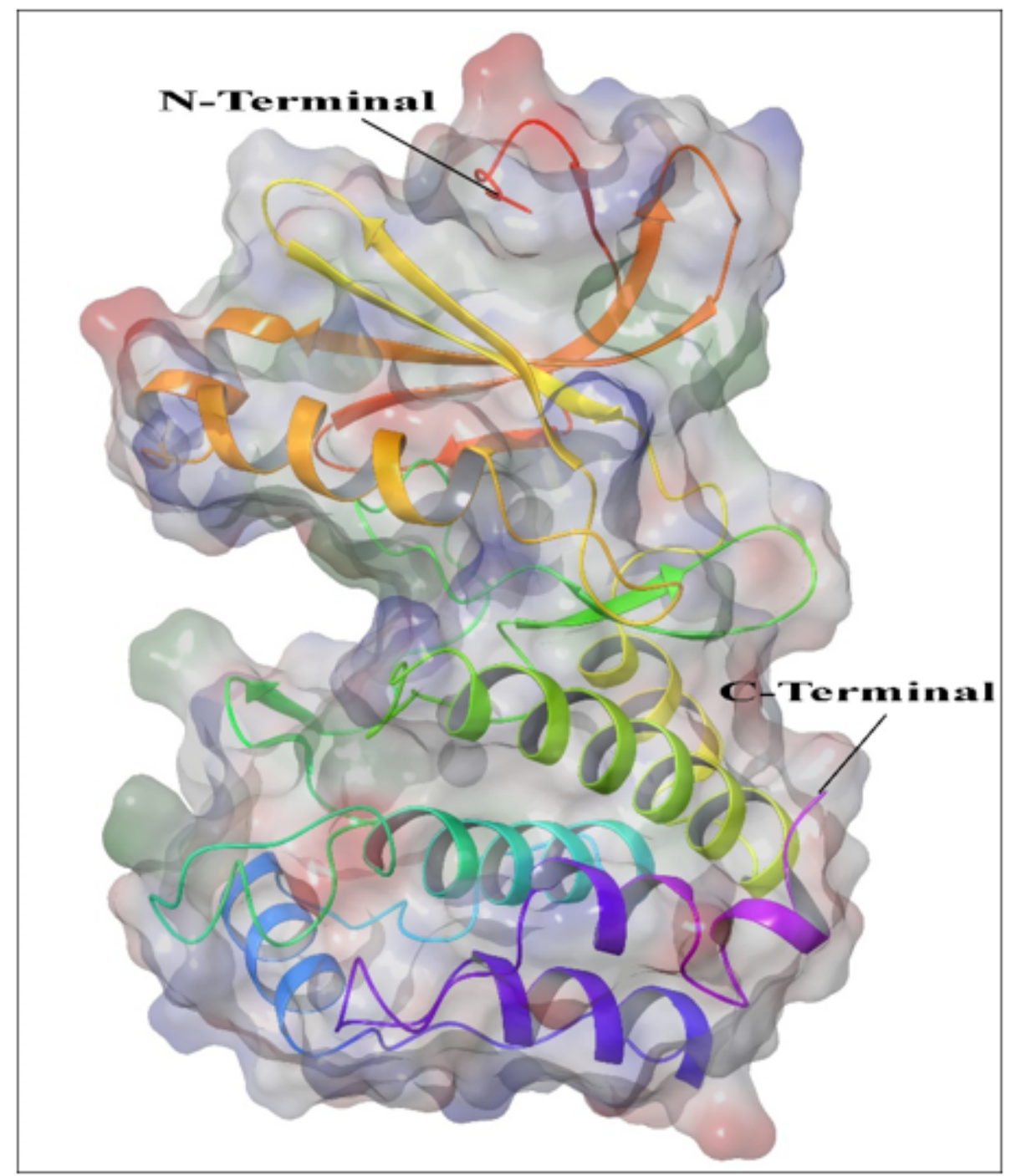

Figure 1

3 D structure of Aurora Kinase A protein retrieved from PDB database. 


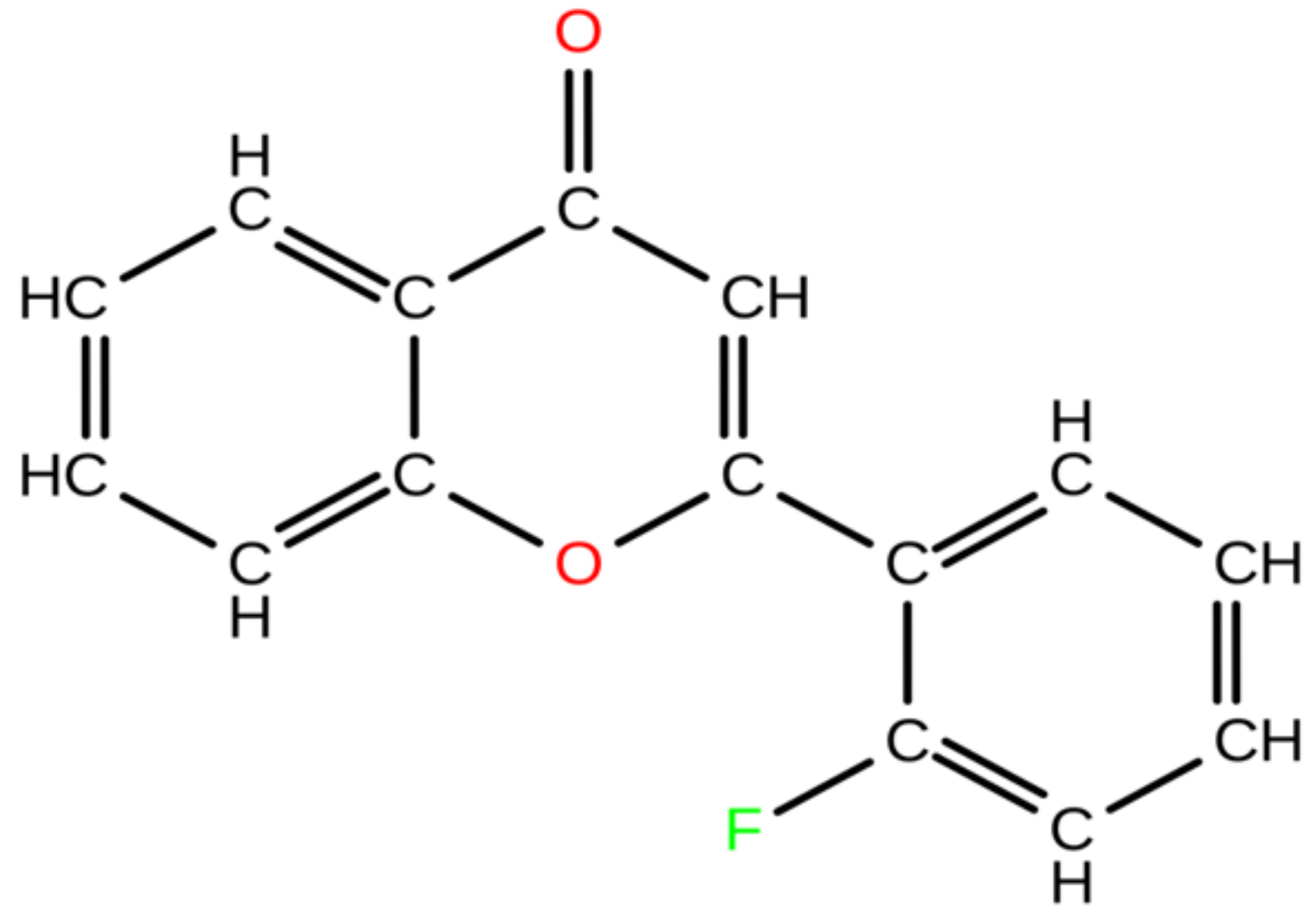

Figure 2

The 2-dimensional (2D) structure of Fluoroflavone. 


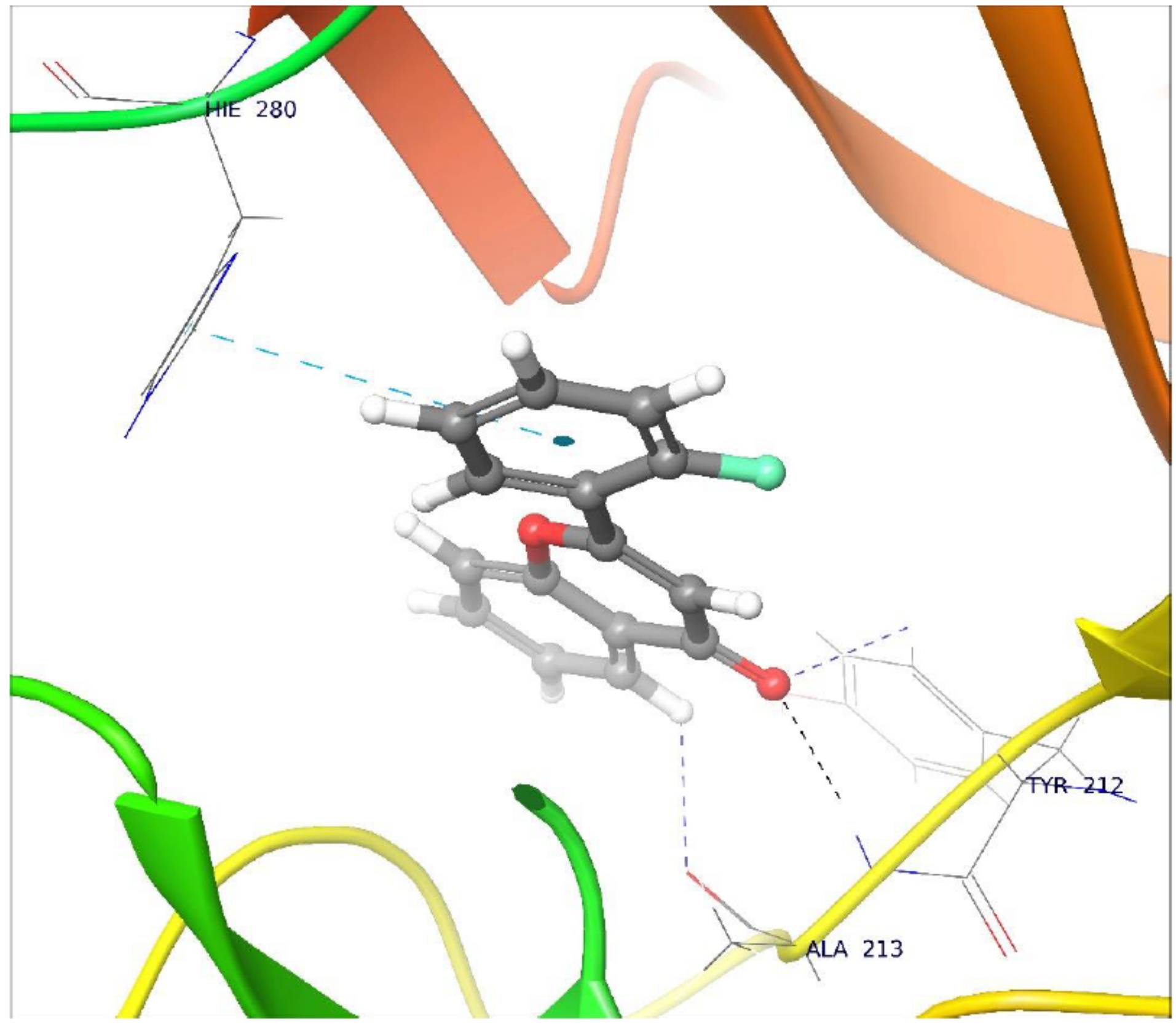

Figure 3

Intermolecular interactions between Fluoroflavone and Aurora Kinase A. Black, blue and cyan coloured dashed lines represent hydrogen bonds, aromatic hydrogen bonds and pi-pi stacking interactions, respectively. Ligand shown in ball and stick model, interacting residues represented in lines and protein backbone shown in ribbon form. 


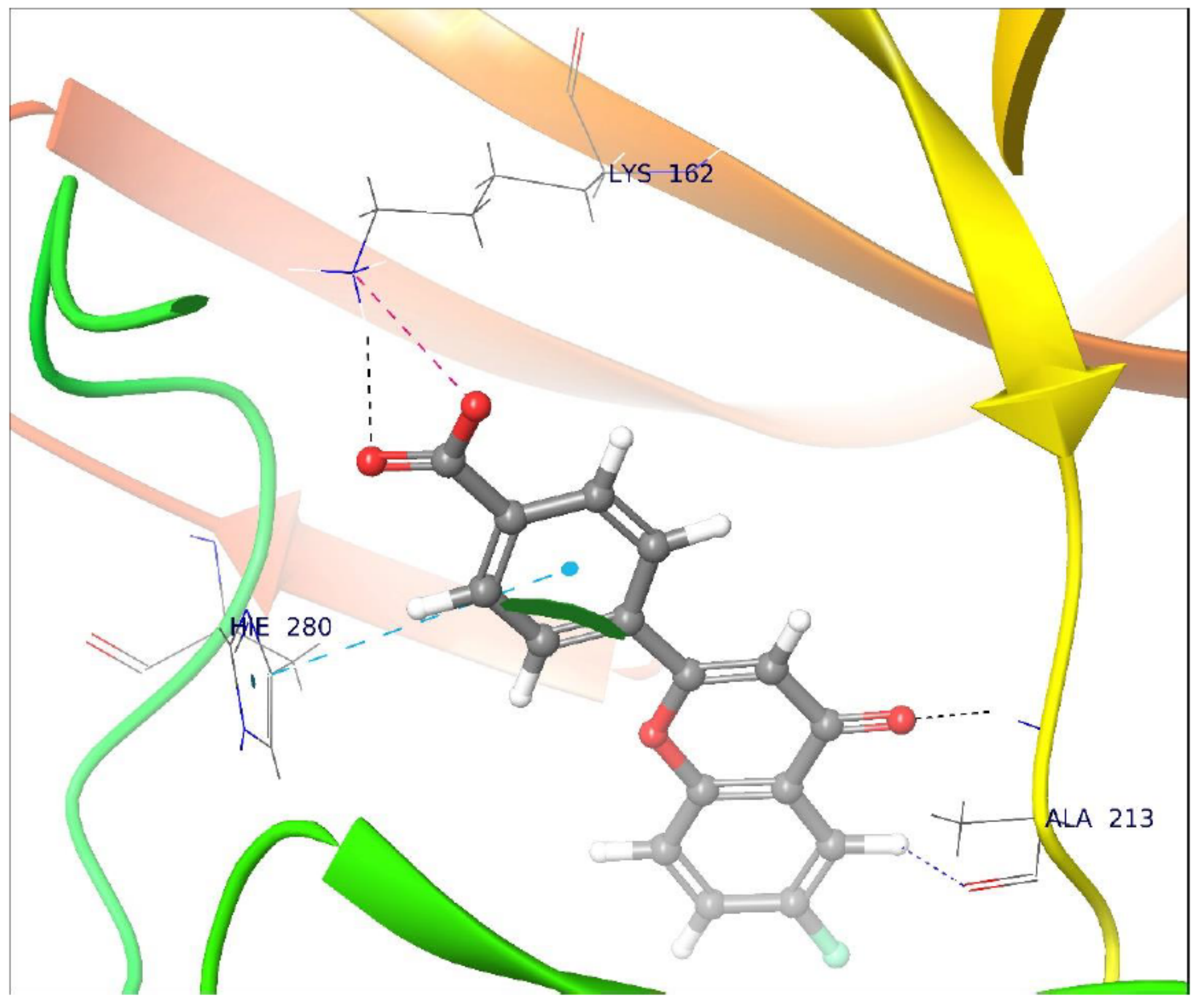

\section{Figure 4}

Intermolecular interactions between Fluoroflavone Analog 1 (CID: 82043699) and Aurora Kinase A. Black, blue, pink and cyan coloured dashed lines represent hydrogen bonds, aromatic hydrogen bonds, salt bridge and pi-pi stacking interactions, respectively. Ligand shown in ball and stick model, interacting residues represented in lines and protein backbone shown in ribbon form. 


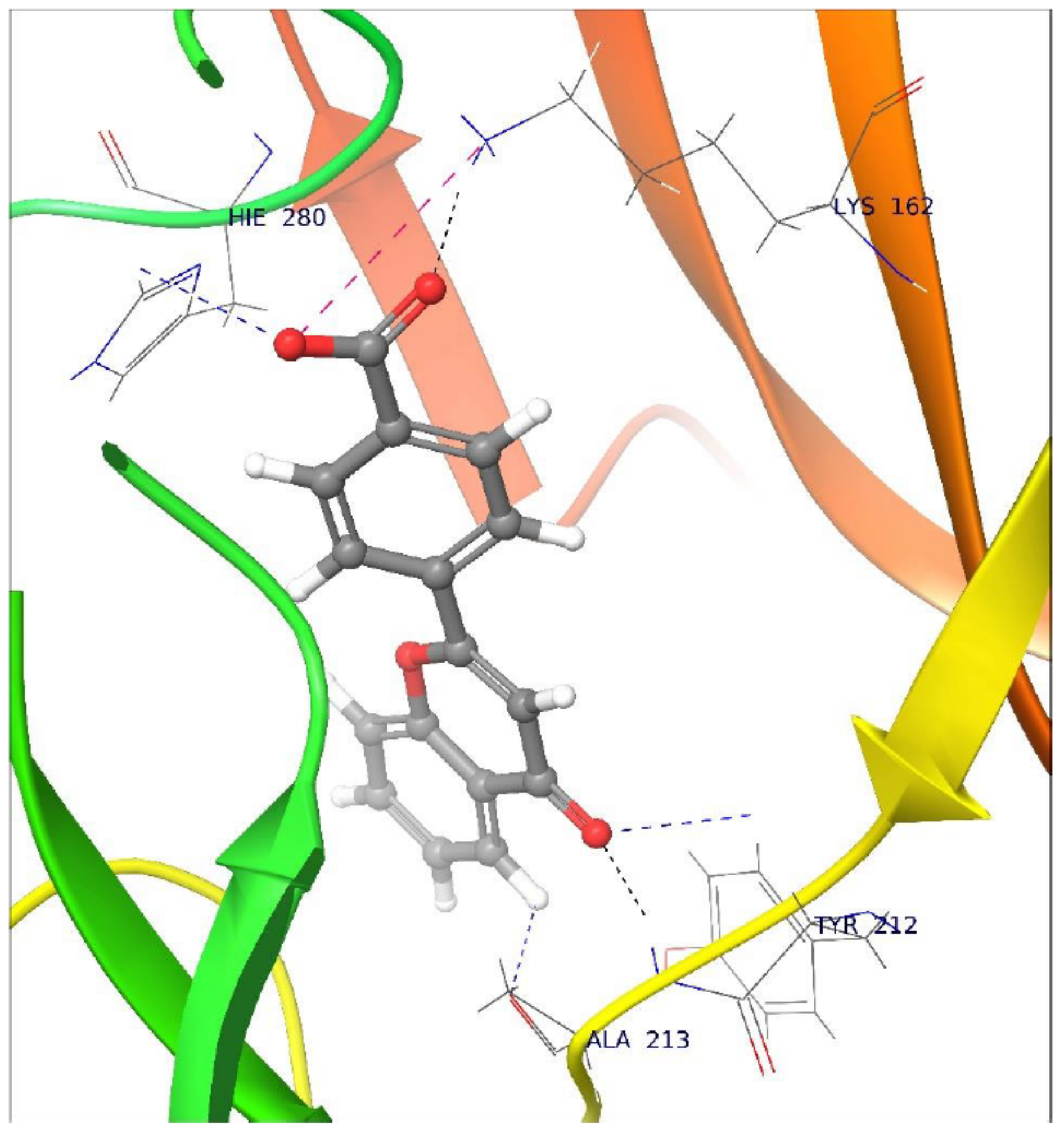

Figure 5

Intermolecular interactions between Fluoroflavone Analog 2 (CID: 7728977) and Aurora Kinase A. Black, blue, pink and cyan coloured dashed lines represent hydrogen bonds, aromatic hydrogen bonds, salt bridge and pi-pi stacking interactions, respectively. Ligand shown in ball and stick model, interacting residues represented in lines and protein backbone shown in ribbon form. 


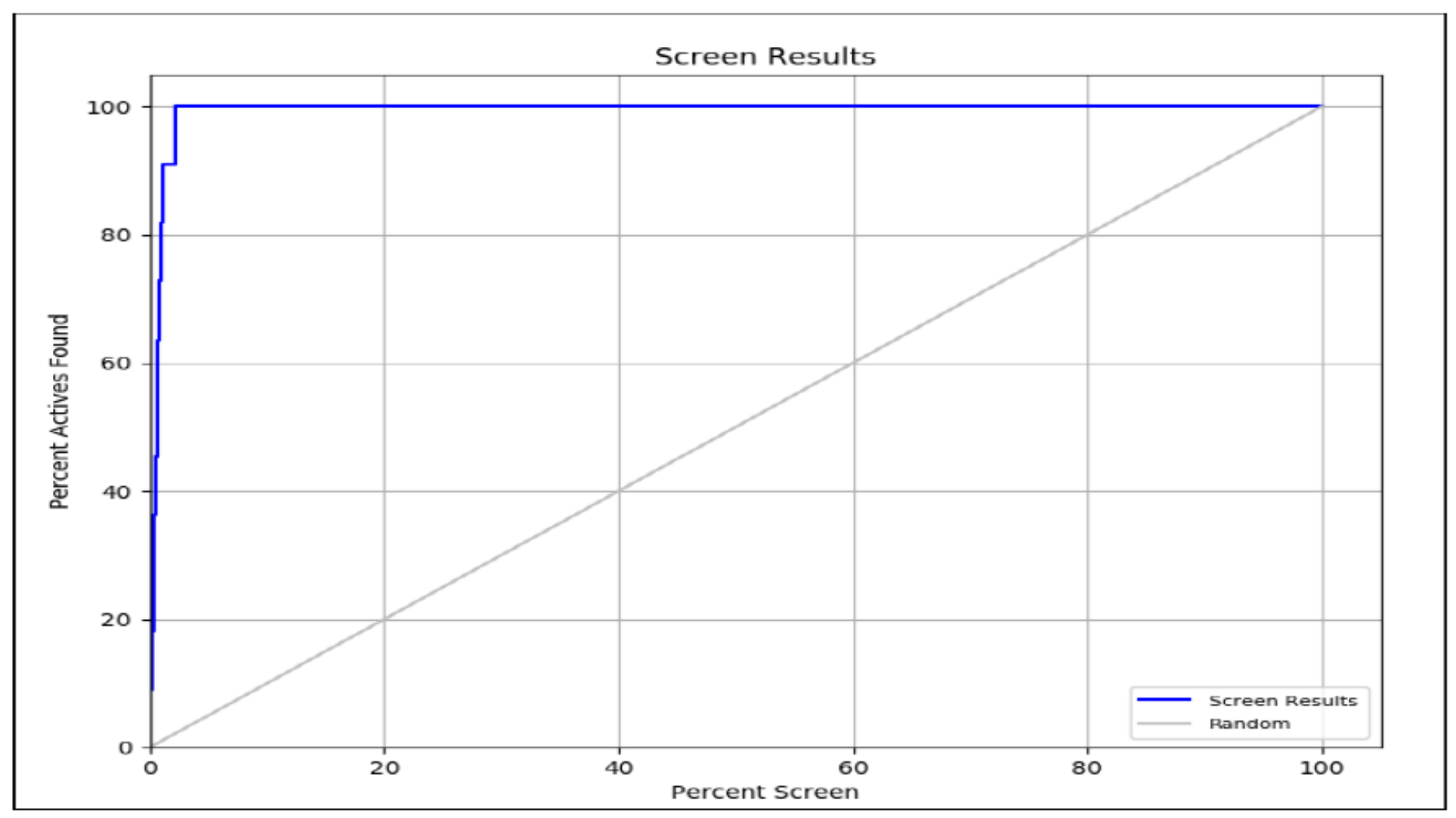

Figure 6

The plot of Receiver Operator Characteristic (ROC) area under the curve. The value is lies between 1 and 0 . The value 1 indicates perfect screen result and $<0.5$ indicates random selection of the screened compounds.

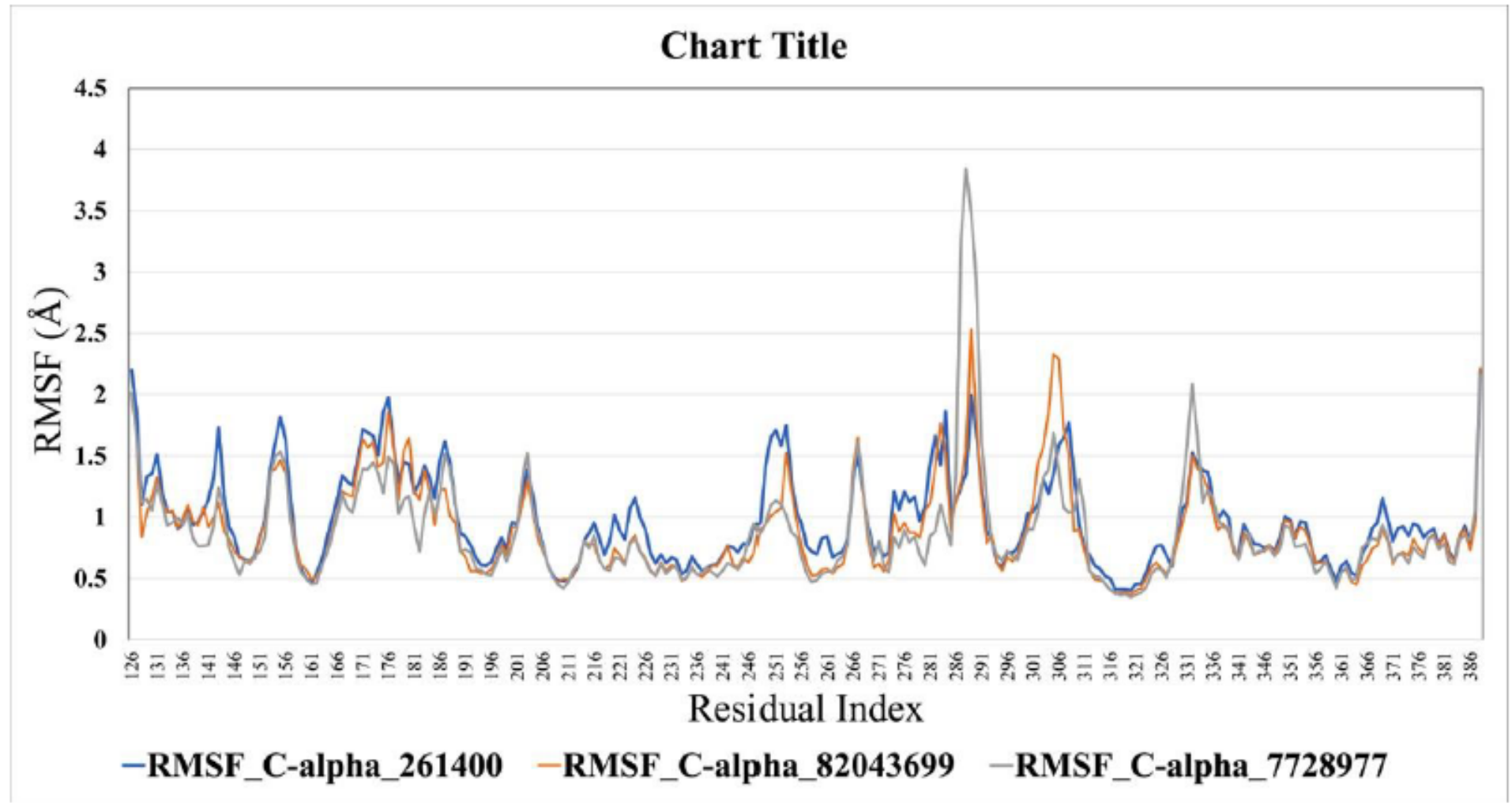

Page $21 / 22$ 


\section{Figure 7}

Time dependence of root mean square fluctuation (RMSFs) of the C-a backbone of Aurora Kinase A after binding with Fluoroflavone, Fluoroflavone Analog 1, and Fluoroflavone Analog 2 with viz. CID: 261400, $82043699,7728977$.

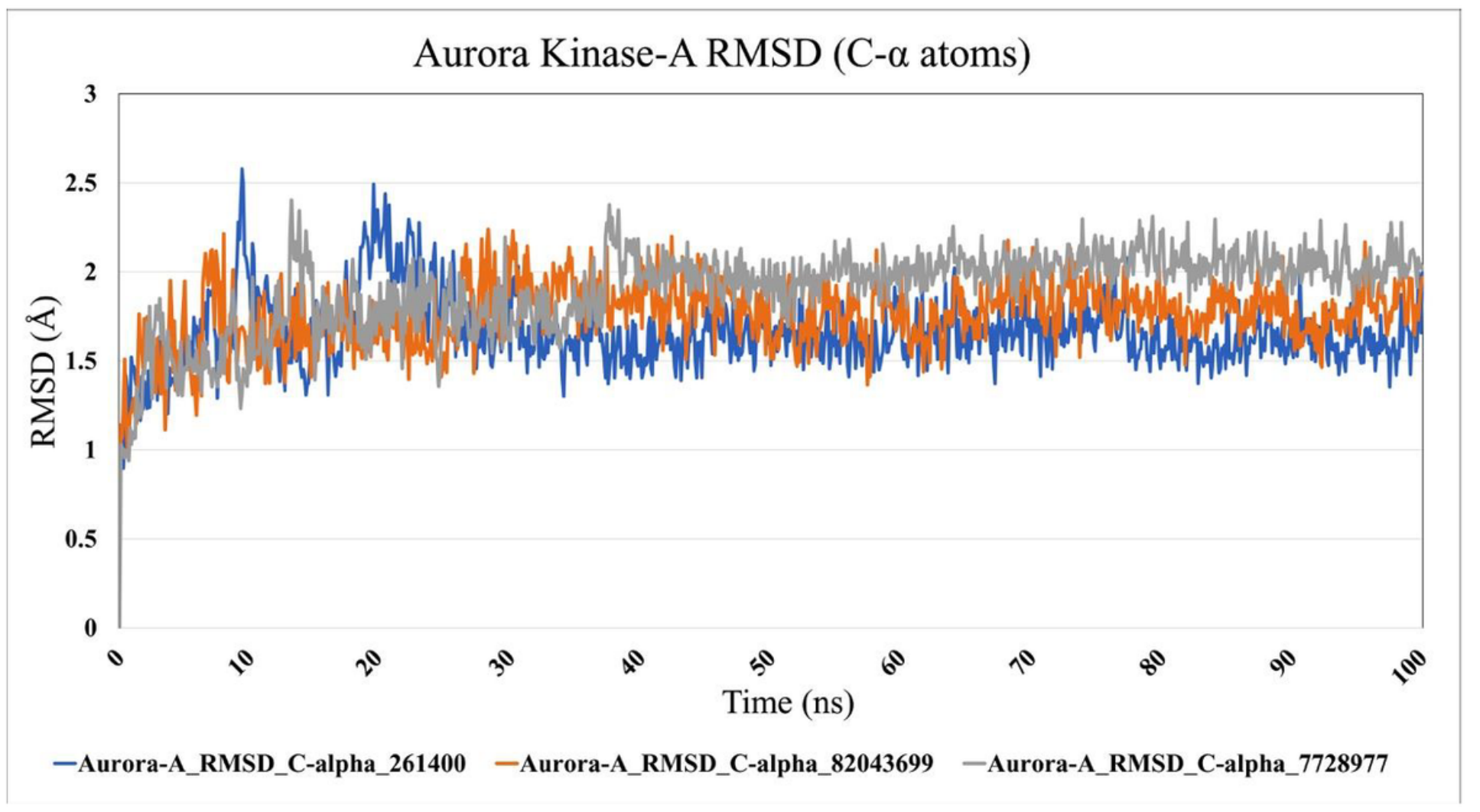

\section{Figure 8}

Time dependence of root mean square deviations (RMSDs) of the C-a backbone of Aurora Kinase A after binding with Fluoroflavone, Fluoroflavone Analog 1, and Fluoroflavone Analog 2 viz. CID: 261400, $82043699,7728977$. 